\title{
Review
}

\section{Recent advances in imaging endogenous or transferred gene expression utilizing radionuclide technologies in living subjects: applications to breast cancer}

\author{
Frank Berger and Sanjiv Sam Gambhir
}

Department of Molecular and Medical Pharmacology, Crump Institute for Molecular Imaging, UCLA School of Medicine, Los Angeles, California, USA

Correspondence: Sanjiv Sam Gambhir, Crump Institute for Molecular Imaging, UCLA School of Medicine, Box 951770, Los Angeles, CA 90095-1770, USA. Tel: +1 310206 1798; fax: +1 310209 4655; e-mail: sgambhir@mednet.ucla.edu

\begin{abstract}
A variety of imaging technologies is being investigated as tools for studying gene expression in living subjects. Two technologies that use radiolabeled isotopes are single photon emission computed tomography (SPECT) and positron emission tomography (PET). A relatively high sensitivity, a full quantitative tomographic capability, and the ability to extend small animal imaging assays directly into human applications characterize radionuclide approaches. Various radiolabeled probes (tracers) can be synthesized to target specific molecules present in breast cancer cells. These include antibodies or ligands to target cell surface receptors, substrates for intracellular enzymes, antisense oligodeoxynucleotide probes for targeting mRNA, probes for targeting intracellular receptors, and probes for genes transferred into the cell. We briefly discuss each of these imaging approaches and focus in detail on imaging reporter genes. In a PET reporter gene system for in vivo reporter gene imaging, the protein products of the reporter genes sequester positron emitting reporter probes. PET subsequently measures the PET reporter gene dependent sequestration of the PET reporter probe in living animals. We describe and review reporter gene approaches using the herpes simplex type 1 virus thymidine kinase and the dopamine type 2 receptor genes. Application of the reporter gene approach to animal models for breast cancer is discussed. Prospects for future applications of the transgene imaging technology in human gene therapy are also discussed. Both SPECT and PET provide unique opportunities to study animal models of breast cancer with direct application to human imaging. Continued development of new technology, probes and assays should help in the better understanding of basic breast cancer biology and in the improved management of breast cancer patients.
\end{abstract}

Keywords: animal models, imaging, microPET, positron emission tomography, single photon emission computed tomography

\section{Radionuclide imaging technologies}

Two major technologies that employ radioactive isotopes (Supplementary Table 1) for imaging molecular events currently exist: SPECT (carried out with a gamma camera), employing isotopes in which a single high energy photon is directly emitted [1]; and PET, in which a positron is emitted from the radioactive isotope [2]. For positron imaging, positrons are emitted from nuclei of proton-rich isotopes,

$\mathrm{CT}=$ computed tomography; $\mathrm{D} 2 \mathrm{R}=$ dopamine type 2 receptor; $\mathrm{ER}=$ estrogen receptor; FDG $=2$-[18F]-2-fluoro-deoxyglucose; FESP $=\left[{ }^{18} \mathrm{~F}\right]-$ fluoroethylspiperone; FIAU = 5-iodo-2'-fluoro-2'-deoxy-1- $\beta$-D-arabinofuranosyl-5-ioduracil; HSV1-tk = herpes simplex type 1 virus thymidine kinase; IRES = internal ribosomal entry sites; MRI = magnetic resonance imaging; PET = positron emission tomography; SPECT = single photon emission computed tomography. 
and eventually interact with electrons. Annihilation occurs, and the mass of the electron and positron is converted into two gamma rays that travel outward from the site of annihilation at $\sim 180^{\circ}$ to one another. Scintillation crystals composed of different materials are used to capture the gamma rays for both SPECT and PET. The collection of many events allows reconstruction of the source of the emissions (Supplementary Figure 1). PET data can be corrected for attenuation, which occurs because some of the gamma rays do not traverse through all of the surrounding tissue. SPECT data is more difficult to correct for attenuation effects. PET is about 10 -fold more sensitive than SPECT, primarily because the SPECT cameras use collimators that reject many of the counts from the source [3]. There are dedicated breast PET imaging systems under development [4] that may prove to be clinically important for breast and/or axillary imaging with improved resolution and/or sensitivity. We have recently developed microPET technology at UCLA, which allows high resolution ( $2 \mathrm{~mm}$ in each axial direction) imaging of small animals such as mice [5] (Supplementary Figure 2).

Although the focus of this review is on radionuclide imaging technologies, it is worth briefly reviewing other imaging modalities and how they contrast to PET and SPECT. Magnetic resonance imaging (MRI) [6] and computed tomography (CT) [7] provide high resolution anatomical imaging that is not available through SPECT and PET. Development of new contrast agents for MRI is allowing the study of molecular events, but these approaches are still in the early developmental stage [8]. Magnetic resonance spectroscopy has also extensively been studied and is reviewed in detail elsewhere [9]. Optical approaches for imaging living small animals have been significantly aided by the use of green fluorescent protein [10] and firefly luciferase [11]. The use of highly sensitive charge-coupled device cameras has provided the ability to detect very low levels of light coming from deep within a living animal. This approach does not produce tomographic images, but provides a rapid, low cost method to detect molecular events using an optical reporter gene.

The advantages of radionuclide technologies and, especially, PET include high sensitivity $\left(\sim 10^{-11} \mathrm{M}\right)$, which allows for detecting low levels of probes accumulated at a given site. Furthermore, because the isotopes available for PET can substitute for naturally occurring atoms in organic molecules, enormous versatility in the study of biochemistry in vivo is possible. The radionuclide approaches do suffer from a lack of anatomical information that can be provided by the other technologies. Multiple efforts are currently underway to build combined MRI/PET and CT/PET systems [12]. We are performing microPET studies in our laboratories and are starting to use microCAT technology (Imtek Inc, Knoxville, TN, USA) [13] to obtain partially registered anatomical information. Clinical scanners that combine CT and PET have also recently become available and should help to improve overall diagnostic capability [14].

\section{Radionuclide imaging probes}

A key advantage of the radionuclide imaging technologies over other imaging approaches is the ability to label almost any chemical species with an isotope of choice. This has allowed the development of hundreds of radioactive imaging probes capable of imaging a variety of molecular events $[2,15]$.

A potentially useful way to discuss the general categories of probes is to start from targets at the cell membrane and to move to targets within the cell. Two major approaches are available for those genes that lead to expression of a protein on the cell surface. These involve antibodies or antibody fragments to target a specific protein, and specific ligands to target receptors on cells.

Antibody fragments are being engineered to achieve rapid targeting and rapid blood clearance [16]. A recent example from our laboratories of an antibody fragment approach targeting carcinoembryonic antigen expression in a mouse tumor xenograft model imaged in microPET is shown in Figure 1. The antibody fragment in this case is labeled with ${ }^{64} \mathrm{Cu}$, which has a half-life of approximately $12 \mathrm{~h}$. It is likely that engineered antibody fragments will provide methods to target many cell surface targets and should continue to be a general class of radiolabeled imaging probe.

Many possibilities for imaging molecular targets exist as one considers targets within a cell. Substrates that are trapped within the cell because they are metabolized by intracellular enzymes can be exploited for imaging purposes. A specific example of this approach is the widely used 2-[18F]-2-fluoro-deoxyglucose (FDG) [15,17], which is actively transported into cells by a glucose membrane transporter. After hexokinase-mediated phosphorylation, phosphorylated FDG (FDG-6-PO ${ }_{4}$ ) cannot be further metabolized in the glucose metabolic pathway and is not able to leave the cell, leading to intracellular trapping of the radiolabeled compound $[2,15]$ Breast carcinomas, like many cancers, show noticeable increased glucose metabolism, leading to a pronounced intracellular accumulation of FDG relative to surrounding tissues. FDG-PET can provide helpful information in the multimodality evaluation of breast lesions, therapy response and extent of metastatic disease. The inability to detect very small tumors $(<4 \mathrm{~mm}$ diameter) and the varying metabolic activity of the different tumor subtypes in breast cancer [18] cause some limitations.

$\left[{ }^{11} \mathrm{C}\right]-$ Methionine, a proliferation marker capable of detecting changes in the amino acid metabolism of tumors, might have an impact in predicting treatment response, but at present very little data in breast cancer patients are available [19]. 
Figure 1

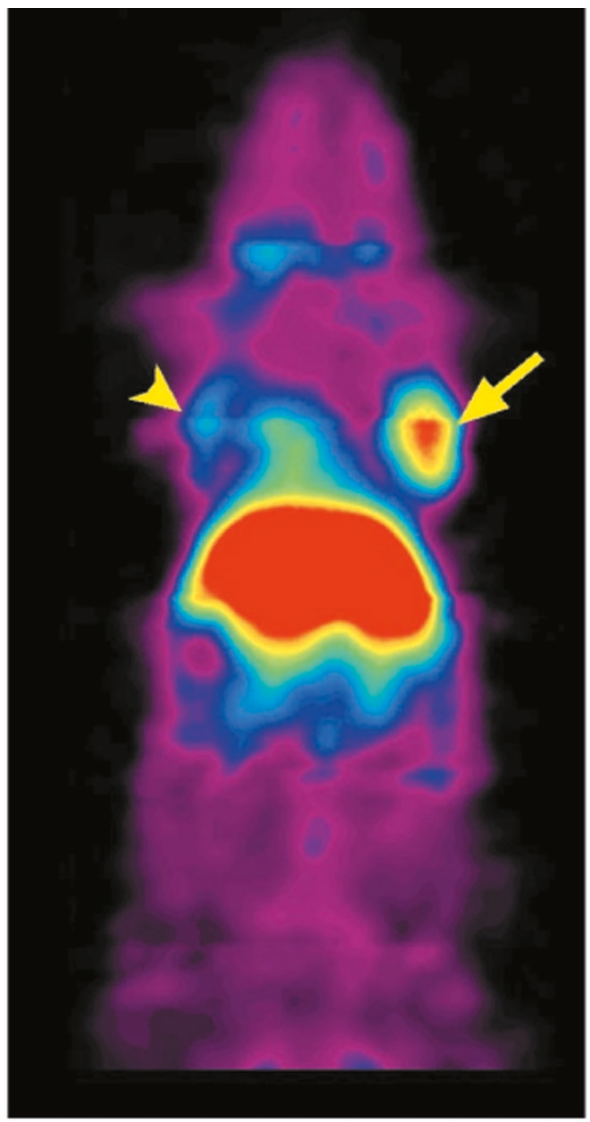

MicroPET imaging of a mouse using a $\left[{ }^{64} \mathrm{Cu}\right]$-labeled antibody fragment targeted against carcinoembryonic antigen (CEA) in a xenograft model. The mouse carried a C6 glioma xenograft on the left shoulder (negative control) and a LS174T xenograft expressing CEA on the right shoulder (arrows). The mouse was injected with $26 \mu \mathrm{Ci}{ }^{64} \mathrm{Cu}$-anti-CEA minibody and imaged at $5 \mathrm{~h}$, with the highest retention in the LS174T tumor (arrow) and liver, and lower retention in the control tumor (arrowhead). The liver signal occurs due to metabolism of the antibody fragment at that site.

In vivo imaging of intracellular receptors has also been reported. The growth of breast epithelial cells is an estrogen-mediated process that depends on estrogen acting through an estrogen receptor (ER) and results in the induction of progestin receptor. The assay of the levels of these two receptors is important because it is considered indicative of the responsiveness of a tumor to hormonal agents [20]. The ER content of breast carcinomas is also known to be an important prognostic indicator. Successful research has focused mainly on imaging of estrogen receptors. $\left[{ }^{18} \mathrm{~F}\right]$-Fluoro-17 $\beta$-estradiol has high affinity for the ER, and an excellent correlation has been noted between the tumoral uptake of this estradiol on PET images and the tumor ER concentration measured in vitro [21].
Figure 2

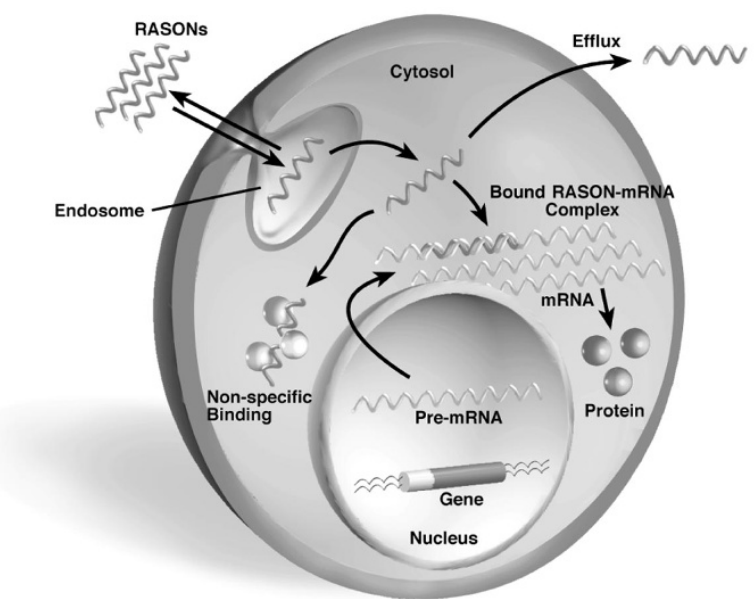

Illustration of antisense hybridization imaging approach. Small radioactive antisense oligodeoxynucleotides (RASONs) are used to target a small portion of a chosen mRNA. If sufficient levels of mRNA are present, it may be possible to retain the RASONs only in those cells expressing the mRNA. Efflux would occur in other cells if nonspecific interactions can be minimized. Because radiolabeling chemistry can be made independent of the RASON sequence, many different types of mRNA can potentially be targeted.

Another important approach that is still in its infancy is the use of small (15-20 bases) antisense oligodeoxynucleotides that target gene expression by specific hybridization to mRNA (Fig. 2). The basis for this approach is rooted in the use of antisense drugs as methods to inhibit translation of mRNA [22]. The ability to image endogenous gene expression using a wide array of radiolabeled probes is quite a general and powerful approach. The rate-limiting step, however, is the ability to radiolabel a specific molecule and not to significantly alter its properties. It would be ideal if there could be a single universal probe that could image a wide variety of molecular events. The antibody fragment approach may satisfy this need for cell membrane targets but not intracellular targets. The antisense approach with mRNA targeting is a potential general approach, but it is currently limited and not likely to image genes with relatively low levels of transcription. If one is able to introduce an imaging or reporter gene into a group of cells, imaging gene expression with a universal probe becomes possible. Several reporter gene approaches for imaging transferred gene expression, including applications to gene therapy models, are now discussed.

\section{Principles of imaging reporter gene expression}

Molecular biologists have long used reporter genes to study promoter/regulatory elements involved in gene expression, inducible promoters to examine the transduction of gene expression, and transgenes containing endogenous promoters fused to a reporter gene to study endogenous gene expression. This is a highly general 
approach because any promoter can be fused to a reporter gene, and detection of a reporter gene product is a way of 'indirectly' following the expression of the gene that the chosen promoter normally regulates. Once a reporter gene driven by a promoter of choice is introduced into the desired tissue, expression of the reporter gene can be monitored by several conventional methods like tissue biopsy, followed by immunohistochemistry. Conventional methods to detect reporter gene expression are, however, limited by their inability to determine 'non-invasively' the locations and magnitude of gene expression in living subjects over time. Approaches using green fluorescent protein [10] and firefly luciferase [11] as reporter genes allow localization of reporter gene expression in some living animals, but monitoring of the detailed location and magnitude of reporter gene expression over time is difficult. Approaches using MRI are reviewed elsewhere [8].

Radionuclide imaging techniques offer the possibility of monitoring the detailed location, magnitude, and time variation of reporter gene expression with high sensitivity for in vivo use in animals and humans [23]. A promoter of choice drives transcription of a reporter gene. The accumulation of the reporter probe is dependent directly on the expression of the reporter gene: the translation of the reporter gene mRNA leads to a protein product that can interact with a reporter probe. This interaction may be based on uptake and enzymatic conversion of the reporter probe via the reporter protein, leading to retention of the metabolite(s) or a receptor ligand based interaction (Fig. 3).

The choice of a promoter for driving reporter gene expression depends on the intended application. Constitutive promoters can be used to produce continuous transcription of the reporter gene. Inducible promoters can be used to provide external control for varying the levels of transcription. To mimic the transcription of some endogenous gene, one can use the identical promoter of the endogenous gene to be imaged. This allows the use of reporter genes to indirectly monitor the expression of an endogenous gene. Two reporter gene approaches are discussed: the herpes simplex type 1 virus thymidine kinase (HSV1-tk) and the dopamine type 2 receptor (D2R). Other approaches have also been developed and are reviewed elsewhere $[24,25]$.

The HSV1-tk gene (HSV1-TK is the corresponding enzyme) has its roots in a therapeutic approach as a 'suicide gene' [25,26]. HSV1-TK, like murine and human TK, phosphorylates thymidine. HSV1-TK, however, phosphorylates acycloguanosines (eg acyclovir, ganciclovir, penciclovir) much more effectively than do mammalian TKs. Cellular enzymes then convert the acycloguanosine monophosphates to diphosphates and triphosphates that, if present in sufficient concentration, kill cells by incorporation as chain-terminating derivatives or by direct inhibition

\section{Figure 3}

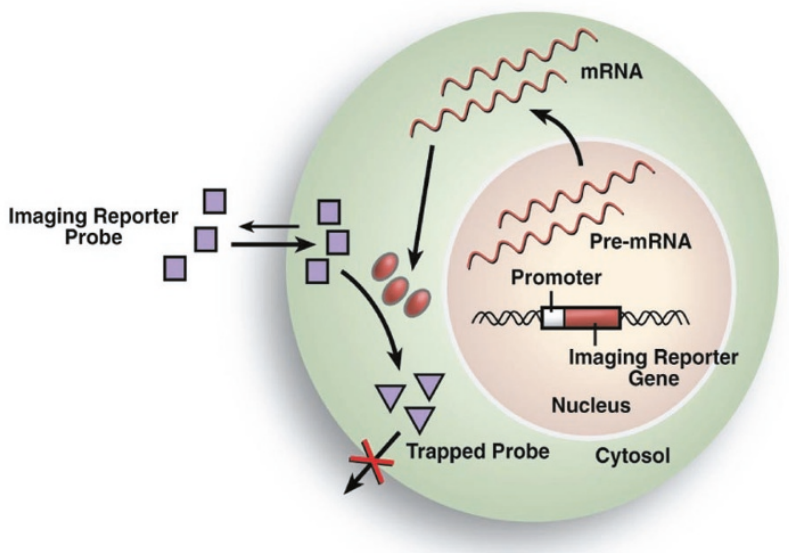

Schematic illustrating a reporter gene approach. An imaging reporter gene has to be introduced into the cell, which is driven by a promoter of choice. Transcription of the imaging reporter gene with subsequent translation of the mRNA leads to an enzyme. This enzyme can selectively trap an imaging reporter probe. The imaging reporter probe will not be trapped in those cells in which there is no expression of the imaging reporter gene. Note that it is also possible for the imaging reporter gene to encode for an intracellular and/or cell surface receptor. This receptor would then bind the imaging reporter probe (a ligand). Levels of the trapped probe can be related to levels of imaging reporter gene expression in either approach.

of DNA polymerase. Because HSV1-TK converts acycloguanosine prodrugs to toxic compounds, this approach is used in cancer gene therapy protocols as a suicide gene approach [26]. The actual approach is to deliver the HSV1-tk gene to tumor cells, then use acycloguanosines to kill the cells expressing HSV1-tk. The relaxed substrate specificity by the viral TK is critical in the approach of HSV1-tk suicide gene therapy, but can also be exploited using the HSV1-tk gene in an imaging approach.

Two main categories of substrates, uracil nucleoside derivatives and acycloguanosine derivatives, have been investigated specifically as reporter probes for imaging HSV1-tk reporter gene expression for SPECT and PET. These reporter probes are transported into cells, and trapped as a result of enzymatic phosphorylation by HSV1-TK. The choice of acycloguanosine derivatives (eg ganciclovir) as potential reporter probes was based on their ability to be radiolabeled with ${ }^{18} \mathrm{~F}$, which has several ideal characteristics as an isotope [27]. The reporter probe is only used in tracer doses (not in pharmacological doses as in suicide therapy), so there are no known problems concerning cell toxicity.

8-[18F]-Fluoroganciclovir has been validated as a reporter probe for HSV1-tk gene expression using a replicationdeficient adenovirus, Ad-CMV-HSV1-tk, encoding HSV1-tk 
Figure 4

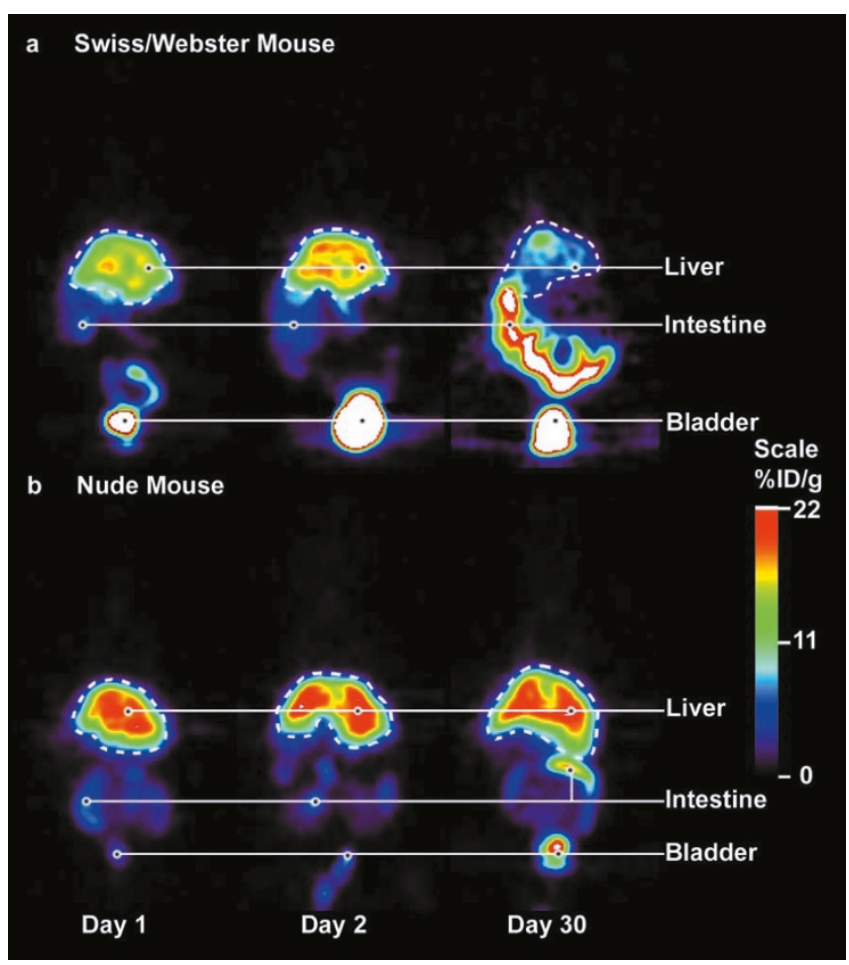

Adenoviral-mediated HSV1-sr39tk gene expression repetitively imaged in two mice. Each mouse was tail-vein injected with $1.0 \times 10^{9} \mathrm{pfu}$ adenovirus in which the HSV1-sr39tk reporter gene is driven by the $\mathrm{CMV}$ promoter. Each mouse was imaged in a microPET system at the days indicated $1 \mathrm{~h}$ after tail-vein injection of $250 \mu \mathrm{Ci}\left[{ }^{18} \mathrm{~F}\right]$-labeled penciclovir molecule (FHBG). The scale represents the accumulation of FHBG measured as percent injected dose per gram of tissue (\%lD/g). (a) The Swiss Webster mouse shows a decrease in gene expression over time, and (b) the nude mouse shows persistence of gene expression in the liver. These differences are likely due to the immune system being fully competent in the Swiss Webster mouse.

expressed under the control of the CMV promoter [28]. Other laboratories are also developing positron-labeled substrates to image the HSV1-tk PET reporter gene in living animals [29]. Radioactive 5-iodo-2'-fluoro-2'-deoxy-1$\beta$-D-arabinofuranosyl-5-ioduracil (FIAU) has been used to image stable HSV1-tk gene expression in transplanted tumors and HSV1-tk genes delivered in retroviral vectors to transplanted tumors. Using [ $\left.{ }^{124}\right]$-labeled FIAU, data similar to that observed with $8-\left[{ }^{18} \mathrm{~F}\right]$-fluoroganciclovir and $8-\left[{ }^{8} \mathrm{~F}\right]$ fluoropenciclovir were obtained in imaging HSV1-tk expression in tumors by PET. Because of its longer half-life (4 days), [ $\left.{ }^{124}\right]$-FIAU may be a useful alternative to image HSV1-tk in some applications in which the background signal needs to be minimized. FIAU can additionally be labeled with single photon emitting iodine, allowing direct imaging with SPECT [30]. Further details of the HSV1-tk reporter gene system and various reporter probes are reviewed elsewhere [25]. We have also recently studied a mutant HSV1-sr39tk reporter gene with a better sensitivity for detecting lower levels of reporter gene expression [31]. Figure 4 presents an example of repetitive microPET imaging of a mutant HSV1-sr39tk reporter gene using a $\left[{ }^{18} \mathrm{~F}\right]$-labeled penciclovir molecule. An adenoviral vector is used to deliver the HSV1-sr39tk reporter gene to the liver. The adenoviral vector targets the liver because of Coxsackie adenoviral receptors present on hepatocytes.

[18F]-Fluoroethylspiperone (FESP) is a positron-labeled analog of the dopamine antagonist spiperone. This tracer was developed to evaluate the level of $\mathrm{D} 2 \mathrm{R}$ in vivo in animals and humans [32]. Endogenous high level D2R expression is limited primarily to the striatum. We have used the D2R gene as a reporter gene and FESP as a reporter probe for imaging gene expression [33]. There is no pharmacological effect of FESP in cells ectopically expressing D2R when FESP is used in small tracer concentrations like in PET imaging. Imaging of both the HSV1-tk and D2R reporter genes in animal models of tumor xenografts had been already successfully performed by our laboratories (Supplementary Figure 3) [34]. Further comparisons of the HSV1-tk and D2R reporter gene systems are provided elsewhere [25].

Potential applications of the reporter gene technologies developed for the study of breast cancer models are numerous. It should now be possible to mark any breast cancer cell line with a reporter gene for subsequent imaging of cell trafficking in living animals. Stable transfection of the reporter gene into a given cell line is possible using plasmids carrying the reporter gene, and antibiotic selection markers or viral vectors with subsequent selection of cell lines stably expressing the reporter gene. Once available, these cells can be directly implanted to study cell growth and trafficking with or without pharmacological intervention. Several models are now under study in which breast cancer metastases can be monitored non-invasively over time. Transgenic mice can also be developed in which a breast tissue specific promoter drives the reporter gene. This allows the study of spontaneous tumors as they develop and metastasize. The reporter genes also provide a unique way to optimize gene therapy through tracking the delivered genes, and are now discussed in detail.

\section{Gene therapy applications}

Several gene therapeutic approaches to breast cancer treatment have been considered. A very important strategy is the attempt to correct or compensate specific genetic defects in breast cancer cells. Such efforts to compensate mutations include ablation of oncogenic products, restoration of receptor expression (eg estrogen receptor expression), alteration of genes involved in the induction of apoptosis, or activation of tumor suppressor genes $[35,36]$. Mutations in the $p 53$ gene are among the very 
common changes in breast cancer [37]. Wild type p53 functions as a tumor suppressor gene in breast cancer cells, as shown by the growth suppression of cells in vitro when transfected with wild type expression vectors [38]. Retroviral delivery of wild type $p 53$ into human breast cancer cells suppresses tumorigenicity in nude mice [38].

Hung et al [39] developed a phase I clinical trial of E1A gene therapy targeting Her-2/neu overexpressing breast and ovarian cancer. HER2/neu overexpression in breast cancer is considered linked with lower response to chemotherapy and unfavorable prognosis. This group identified a viral transcriptional regulator, the adenovirus type $5 E 1 A$, that can repress HER-2/neu overexpression. Expression of E1A in HER-2/neu overexpressing cancer cells resulted in downregulation of HER-2/neu in vitro, which reversed the malignant phenotype and restored sensitivity to chemotherapeutic agents. Preclinical in vivo studies showed that the E1A gene was able to function therapeutically as a tumor suppressor gene in breast cancer xenograft models. In the phase I clinical trial, Hung et al showed that the E1A gene can be delivered to cancer cells by a cationic liposomal delivery system. HER2/neu was downregulated and the E1A gene induced apoptosis. A general drawback of the mutation compensation approach is that transfection of most of the tumor cells is needed to obtain a clinical effect, because intracellular responses are being modulated without effect on surrounding non-transfected cells.

Approaches such as molecular chemotherapy (shows a regional 'bystander effect') or immune therapy (has the potential to generate a systemic response) hold even more promise for becoming the breast cancer therapies of the future [35]. Molecular chemotherapy aims to increase the sensitivity of the tumor cells to chemotherapy or to find more specific ways of drug delivery to tumor cells [40]. The most studied system to accomplish cell killing uses the already discussed TK gene from the herpes simplex virus and gangiclovir as a prodrug.

Tumor specificity of molecular chemotherapy can be enhanced through the use of transductional or transcriptional targeting. Transductional targeting directs viral gene delivery vectors specifically to tumor cells, which express unique epitopes like erbB2 or the mucin protein core. Transcriptional targeting may be accomplished through the use of breast or tumor specific promoters such as Muc-1, HER-2, Myc and others [40].

Imaging modalities to verify the location, magnitude and duration of transgene expression are highly recommended in all these approaches, and will be very useful in attempts to optimize gene therapy protocols in general and time of prodrug application in particular.
A third strategy, genetic immunotherapy [35], aims to augment the specificity and magnitude of the immune response against tumor-associated antigens. These therapies include passive and active immunization, introduction of cytokines, and expression of $\mathrm{T}$ cell costimulatory molecules [36]. An advantage of this approach is not having to introduce the therapeutic gene into all metastatic sites. The use of cytokines for immunotherapy has been limited by the toxicities associated with systemic administration of the proteins. Recombinant adenoviral vectors containing various cytokine genes have been used to deliver high local concentrations of the cytokines intratumorally. In a subcutaneous model of metastatic breast cancer in transgenic mice, direct intratumoral injection of an adenoviral vector expressing IL-12 resulted in tumor regression in $75 \%$ of the treated animals [41]. Adenoviral-mediated gene delivery of IL-2 was recently evaluated in a phase I clinical trial in metastatic breast cancer and melanoma patients, showing the safety of this approach [42].

Given the magnitude of breast cancer as a clinical problem, however, few gene therapy clinical trials have until now been initiated. The exceptional heterogeneous molecular biology of this cancer, production of immunosuppressive factors, and the widespread nature of the disease are three examples of the difficulties faced in breast cancer gene therapy [36]. Recent advances in understanding the molecular cell biology underlying breast cancer nevertheless revealed several potentially clinically useful gene therapy approaches for the near future.

Non-invasive imaging of transgene expression will be of great benefit in assessing organ tissue specificity as well as level and duration of transgene expression in vivo, and could therefore be very useful in validation of new delivery systems as well as in monitoring clinical gene therapy trials. Most therapeutic transgenes do not lend themselves to direct imaging of the transgene product. Most therapeutic transgene products lack appropriate ligands or probes that can be radiolabeled and used to generate images that define the magnitude of transgene expression. It would also be very difficult to develop and validate new ligands and probes for each therapeutic transgene. Alternatively, it is reasonable to develop and validate indirect imaging strategies using a reporter gene in combination with a therapeutic gene.

Three strategies can potentially be used for imaging gene expression for gene therapy [25]. One strategy uses a fusion gene containing cDNA from both the reporter and therapeutic gene. The second strategy uses a cis-linked reporter gene in which a single mRNA is transcribed from both genes [43]. In the final strategy, the same amount of two separate vectors can be injected. One vector is used to transfer the therapeutic gene, and the second delivers the reporter gene. The injected vectors are identical apart 
from this genetic difference, and the same promoter drives both genes. The expression of the reporter gene reflects, at the macroscopic tissue level, at least in some diagnostic situations, the expression of the therapeutic gene [44]. All three strategies are based on demonstrating a proportional and constant relationship in the coexpression of two transgenes over a wide range of expression levels. The advantage of these paradigms is that they can potentially be applied to any gene combination. Strict coexpression of two proteins in equimolar amounts can only be achieved by a fusion gene encoding both gene products. However, fusion proteins may not yield functional activity, may not localize in the appropriate subcellular compartment, or may induce immunogenic reactions; therefore, this approach is not ideal for in vivo studies. The proportional expression of two cis-linked genes is possible using an internal ribosomal entry sites (IRES) element within a single bicistronic transcription unit. The IRES element enables translation initiation within the bicistronic mRNA, thus permitting gene coexpression by cap-dependent translation of the first cistron and cap-independent, IRESmediated translation of the second cistron. It will eventually be important to assess whether IRES-based vectors are reliable indicators of transgene coexpression in different tissues, taking into account the half-life of each encoded protein. Whether the genes located upstream and downstream are equally expressed for a given application also has to be investigated. An example of using a bicistronic system to image gene expression in a tumor model is available elsewhere (Supplementary Figure 4) [43]. This work should help form the basis for extending this approach into gene therapy trials.

\section{Future prospects}

The future potential of radionuclide assays with SPECT and, particularly, PET imaging is quite promising. As small animal imaging technologies continue to spread to major research centers and the development of general probes expands, animal research should be significantly accelerated. The study of breast cancer cell trafficking and basic tumor biology in xenograft and transgenic models should be possible. Optimization of gene therapy should also be possible. The results from animal models should be rapidly translated into human applications because of a rapidly growing base of imaging scanners, which should lead to improved diagnosis and management of breast cancer patients.

\section{Acknowledgements}

The authors would like to thank Drs H Herschman, M Phelps, JR Barrio, S Cherry, N Satyamurthy, T Toyokuni, L Wu and A Wu for their collaborative efforts in much of the work described in the paper. We would also like to thank the many undergraduate and graduate students as well as post-doctoral fellows who helped with some of the studies reviewed in this paper

This work is supported in part by US Army DAMD 17-98-1-8179, Department of Energy DE-FC03-87ER60615, NIH RO1 CA82214-01, and $\mathrm{NIH} \mathrm{CA86306;} \mathrm{Frank} \mathrm{Berger} \mathrm{is} \mathrm{supported} \mathrm{by} \mathrm{the} \mathrm{Deutsche}$ Forschungsgemeinschaft.

\section{References}

1. Budinger TF: Critical review of PET, SPECT and neuroreceptor studies in schizophrenia. J Neural Transm 1992, 36(suppl): 3-12.

2. Phelps ME: Inaugural article: positron emission tomography provides molecular imaging of biological processes. Proc Natl Acad Sci USA 2000, 97:9226-9233.

3. Fleming JS, Alaamer AS: Influence of collimator characteristics on quantification in SPECT. J Nucl Med 1996, 37:1832-1836.

4. Freifelder R, Karp JS: Dedicated PET scanners for breast imaging. Phys Med Biol 1997, 42:2463-2480.

5. Cherry SR, Shao Y, Silverman RW, Meadors K, Siegel S, Chatziioannou A, Young JW, Jones WF, Moyers JC, Newport D, Boutefnouchet A, Farquhar TH, Andreaco M, Paulus MJ, Binkley DM, Nutt R, Phelps ME: MicroPET: a high resolution PET scanner for imaging small animals. IEEE Trans Nucl Sci 1997, 44: $1161-1166$.

6. Jackson EF, Ginsberg LE, Schomer DF, Leeds NE: A review of MRI pulse sequences and techniques in neuroimaging. Surg Neurol 1997, 47:185-199.

7. Hopper KD, Singapuri K, Finkel A: Body $\mathbf{C T}$ and oncologic imaging. Radiology 2000, 215:27-40.

8. Bogdanov A, Weissleder R: The development of in vivo imaging systems to study gene expression. Trends Biotechnol 1998, 16:5-10.

9. Rudin M, Beckmann N, Porszasz R, Reese T, Bochelen D, Sauter A: In vivo magnetic resonance methods in pharmaceutical research: current status and perspectives. NMR Biomed 1999, 12:69-97.

10. Yang M, Baranov E, Moossa AR, Penman S, Hoffman RM: Visualizing gene expression by whole-body fluorescence imaging. Proc Natl Acad Sci USA 2000, 97:12278-12282.

11. Contag PR, Olomu IN, Stevenson DK, Contag CH: Bioluminescent indicators in living mammals. Nat Med 1998, 4:245-247.

12. Shao $Y$, Cherry SR, Farahani K, Slates R, Silverman RW, Meadors K, Bowery A, Siegel S, Marsden PK, Garlick PB: Development of a PET detector system compatible with MRI/NMR systems. IEEE Trans Nucl Sci 1997, 44:1167-1171.

13. Paulus MJ, Gleason SS, Kennel SJ, Hunsicker PR, Johnson DK: High resolution X-ray computed tomography: an emerging tool for small animal cancer research. Neoplasia 2000, 2:62-70.

14. Beyer T, Townsend DW, Brun T, Kinahan PE, Charron M, Roddy R, Jerin J, Young J, Byars L, Nutt R: A combined PET/CT scanner for clinical oncology. J Nucl Med 2000, 41:1369-1379.

15. Phelps M: PET: the merging of biology and imaging into molecular imaging. J Nucl Med 2000, 41:661-681.

16. Wu AM: Designer genes: recombinant antibody fragments for biological imaging. Q J Nucl Med 2000, 44:268-283.

17. Higashi K, Clavo AC, Wahl RL: Does FDG uptake measure the proliferative activity of human cancer cells? In vitro comparison with DNA flow cytometry and tritiated thymidine uptake. $J$ Nucl Med 1993, 34:414-418.

18. Avril N, Rose CA, Schelling M, Dose J, Kuhn W, Bense S, Weber W, Ziegler S, Graeff $H$, Schwaiger M: Breast imaging with positron emission tomography and fluorine-18 fluorodeoxyglucose: use and limitations. J Clin Oncol 2000, 18:3495-3502.

19. Huovinen R, Leskinen-Kallio S, Nagren K, Lehikoinen P, Ruotsalainen $U$, Teras M: Carbon-11-methionine and PET in evaluation of treatment response of breast cancer. $\mathrm{Br} J$ Cancer 1993, 67:787-791.

20. Varagnolo L, Stokkel MP, Mazzi U, Pauwels EK: ${ }^{18} \mathrm{~F}$-Labeled radiopharmaceuticals for PET in oncology, excluding FDG. Nucl Med Biol 2000, 27:103-112.

21. Mintun MA, Welch MJ, Siegel BA, Mathias CJ, Brodack JW, McGuire $\mathrm{AH}$, Katzenellenbogen JA: Breast cancer: PET imaging of estrogen receptors. Radiology 1988, 169:45-48.

22. Crooke ST, Lebleu B: Antisense Research and Applications. Ann Arbor: CRC Press, Inc., 1993:579.

23. Gambhir SS, Barrio JR, Herschman HR, Phelps ME: Imaging gene expression: principles and assays. J Nucl Cardiol 1999, 6:219-233.

24. Rogers BE, Zinn KR, Buchsbaum DJ: Gene transfer strategies for improving radiolabeled peptide imaging and therapy. $\mathrm{Q} J$ Nucl Med 2000, 44:208-223.

25. Gambhir SS, Herschman HR, Cherry SR, Barrio JR, Satyamurthy N, Toyokuni T, Phelps ME, Larson SM, Balatoni J, Finn R, Sadelain M, 
Tjuvajev J, Blasberg R: Imaging transgene expression with radionuclide imaging technologies. Neoplasia 2000, 2:118-138.

26. Moolten FL: Suicide genes for cancer therapy. Sci Med 1997, 4:16-25.

27. Gambhir SS, Barrio JR, Herschman HR, Phelps ME: Assays for noninvasive imaging of reporter gene expression. Nucl Med Biol 1999, 26:481-490.

28. Gambhir SS, Barrio J, Wu L, lyer M, Namavari M, Satyamurthy $N$, Bauer E, Parrish C, MacLaren D, Borghei A, Berk A, Cherry S, Phelps $\mathrm{ME}$, Herschman $\mathrm{H}$ : Imaging of adenoviral directed herpes simplex virus type 1 thymidine kinase gene expression in mice with ganciclovir. J Nucl Med 1998, 39:2003-2011.

29. Tjuvajev JG, Joshi R, Lindsley L, Balatoni J, Finn R, Larson S, Sadelain $M$, Blasberg R: Noninvasive imaging of HSV1-tk marker gene with FIAU for monitoring transfer and expression of other therapeutic genes by multi-gene delivery vectors [abstract]. J Nucl Med 1998, 39:130P.

30. Tjuvajev JG, Chen SH, Joshi A, Joshi R, Guo ZS, Balatoni J, Ballon D, Koutcher J, Finn R, Woo SL, Blasberg RG: Imaging adenoviral-mediated herpes virus thymidine kinase gene transfer expression in vivo. Cancer Res 1999, 59:5186-5193.

31. Gambhir SS, Bauer E, Black ME, Liang Q, Kokoris MS, Barrio JR, lyer M, Namavari M, Satyamurthy N, Green LA, Nguyen K, Cherry SR, Phelps ME, Herschman HR: A mutant herpes simplex virus type 1 thymidine kinase reporter gene shows improved sensitivity for imaging reporter gene expression with positron emission tomography. Proc Natl Acad Sci USA 2000, 97: 2785-2790.

32. Barrio JR, Huang SC, Phelps ME: Biological imaging and the molecular basis of dopaminergic diseases. Biochem Pharmacol 1997, 54:341-348.

33. MacLaren DC, Gambhir SS, Satyamurthy N, Barrio JR, Sharfstein S, Toyokuni T, Wu L, Berk AJ, Cherry SR, Phelps ME, Herschman HR: Repetitive, non-invasive imaging of the dopamine $D_{2}$ receptor as a reporter gene in living animals. Gene Ther 1999, 6:785-791.

34. lyer $M$, Bauer $E$, Barrio J, lyer $M$, Namavari $M$, Satyamurthy $N$, Green LA, Nguyen K, Phelps ME, Herschman HR, Gambhir SS: 8-[18F]Fluoropenciclovir: an improved reporter probe for imaging HSV1-tk reporter gene expression in vivo using positron emission tomography. J Nucl Med (in press).

35. Ruppert JMWM, Rosenfeld M, Grushcow J, Bilbao G, Curiel DT, Strong TV: Gene therapy strategies for carcinoma of the breast. Breast Cancer Res Treat 1997, 44:93-114.

36. Boxhorn HK, Eck SL: Gene therapy for breast cancer. Hematol Oncol Clin North Am 1998, 12:665-675.

37. Phillips $\mathrm{H}$ : The role of the $p 53$ tumour suppressor gene in human breast cancer. Clin Oncol (R Coll Radiol) 1999, 11: 148-155.

38. Wang NP, To H, Lee WH, Lee EY: Tumor suppressor activity of RB and p53 genes in human breast carcinoma cells. Oncogene 1993, 8:279-288.

39. Hung MC, Hortobagyi GN, Ueno NT: Development of clinical trial of E1A gene therapy targeting HER-2/neu-overexpressing breast and ovarian cancer. Adv Exp Med Biol 2000, 465:171-180.

40. Patterson A, Harris AL: Molecular chemotherapy for breast cancer. Drugs Aging 1999, 14:75-90.

41. Bramson JL, Hitt M, Addison CL, Muller WJ, Gauldie J, Graham FL: Direct intratumoral injection of an adenovirus expressing interleukin-12 induces regression and long-lasting immunity that is associated with highly localized expression of interleukin-12. Hum Gene Ther 1996, 7:1995-2002.

42. Stewart AK, Lassam NJ, Quirt IC, Bailey DJ, Rotstein LE, Krajden M, Dessureault S, Gallinger S, Cappe D, Wan Y, Addison CL, Moen RC, Gauldie J, Graham FL: Adenovector-mediated gene delivery of interleukin-2 in metastatic breast cancer and melanoma: results of a phase 1 clinical trial. Gene Ther 1999, 6:350-363.

43. Yu Y, Annala AJ, Barrio JR, Toyokuni T, Satyamurthy N, Namavari M, Cherry SR, Phelps ME, Herschman HR, Gambhir SS: Quantification of target gene expression by imaging reporter gene expression in living animals. Nat Med 2000, 6:933-937.

44. Yaghoubi S, Liang $Q$, Barrio JR, Namavari M, Satyamurthy $S$, Toyokuni T, Black M, Phelps ME, Herschman HR, Gambhir SS: Assessment of gene expression utilizing two adenoviral vectors and positron emission tomography [abstract]. FASEB
2000, 14:A568.

\section{Supplementary Table 1}

Radionuclides of interest for positron emission tomography (PET) and single photon emission computed tomography (SPECT) studies

\begin{tabular}{lcc}
\hline Radionuclide & Half-life & Type \\
\hline${ }^{11} \mathrm{C}$ & $20.3 \mathrm{~min}$ & PET \\
${ }^{13 \mathrm{~N}}$ & $9.97 \mathrm{~min}$ & PET \\
${ }^{15 \mathrm{O}}$ & $122 \mathrm{~s}$ & PET \\
${ }^{18} \mathrm{~F}$ & $109.8 \mathrm{~min}$ & PET \\
${ }^{62} \mathrm{Cu}$ & $9.74 \mathrm{~min}$ & PET \\
${ }^{64} \mathrm{Cu}$ & $12.7 \mathrm{~h}$ & PET \\
${ }^{68} \mathrm{Ga}$ & $68.1 \mathrm{~min}$ & PET \\
${ }^{76} \mathrm{Br}$ & $16.1 \mathrm{~h}$ & PET \\
${ }^{124 \mathrm{I}}$ & 4.17 days & PET \\
${ }^{131} \mathrm{I}$ & 8.0 days & SPECT \\
${ }^{123 \mathrm{I}}$ & $13.2 \mathrm{~h}$ & SPECT \\
${ }^{111} \mathrm{ln}$ & 2.8 days & SPECT \\
${ }^{67} \mathrm{Ga}$ & $78.3 \mathrm{~h}$ & SPECT \\
${ }^{99 \mathrm{~m} T \mathrm{C}}$ & $6.0 \mathrm{~h}$ & SPECT \\
${ }^{201} \mathrm{TI}$ & $73.1 \mathrm{~h}$ & SPECT \\
${ }^{127} \mathrm{Xe}$ & 36 days & SPECT \\
${ }^{133 \mathrm{Xe}}$ & 5.2 days & SPECT \\
\hline
\end{tabular}




\section{Supplementary Figure 1}

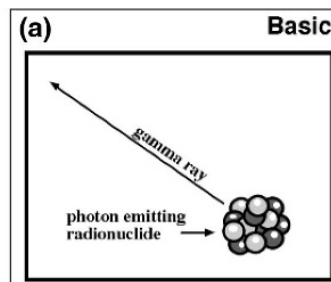

Photon emission

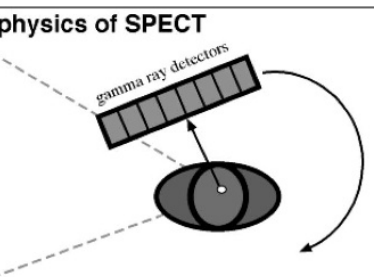

SPECT scanner (b)

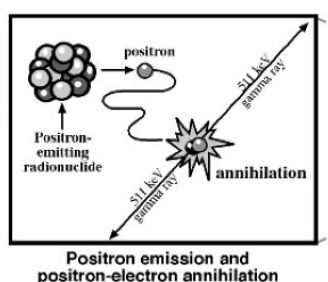

Basic physics of PET

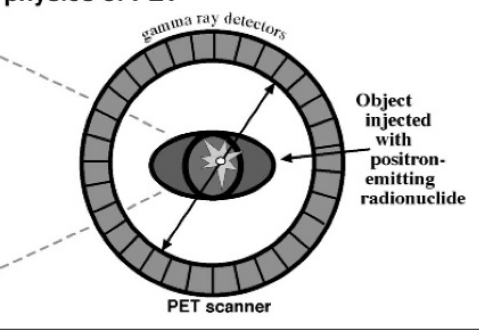

Schematic illustrating single photon emission computed tomography (SPECT) and positron emission tomography (PET). (a) In SPECT, a single photon is produced as the isotope decays and this single photon must be detected through rotating detectors using a collimator. (b) In PET, annihilation eventually occurs with the positron and electron to produce two high energy (511 keV) gamma rays at $\sim 180^{\circ}$ that are detected using a circular ring of detectors.

\section{Supplementary material}

\section{Supplementary Figure 3}

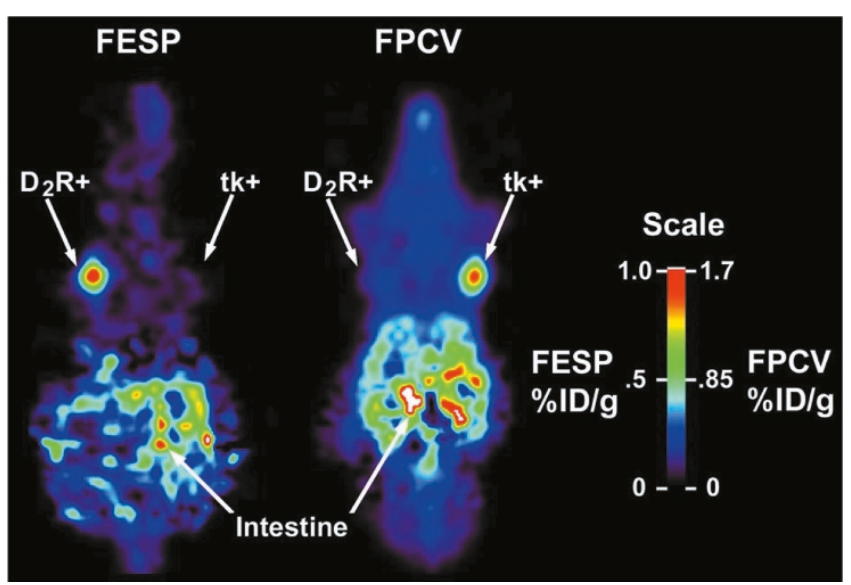

Imaging of two reporter genes in the same mouse. A nude mouse carrying two tumors (left, dopamine 2 receptor [D2R]; right, herpes simplex virus type 1 thymidine kinase [HSV1-tk]) was imaged in a microPET system with tail-vein injection of $\sim 250 \mu \mathrm{Ci}\left[{ }^{18} \mathrm{~F}\right]$ fluoroethylspiperone (FESP) and, 24 hours later, with $\sim 250 \mu \mathrm{Ci} 8$ $\left[{ }^{18} \mathrm{~F}\right]$-fluoropenciclovir (FPCV). Imaging began 1 hour after injection of each tracer. These results show the ability to image both the D2R and the HSV1-tk reporter genes in the same living mouse with microPET. The scale represents the accumulation of FESP and FPCV measured as percent injected dose per gram of tissue $(\% \mathrm{ID} / \mathrm{g})$.

\section{Supplementary Figure 2}

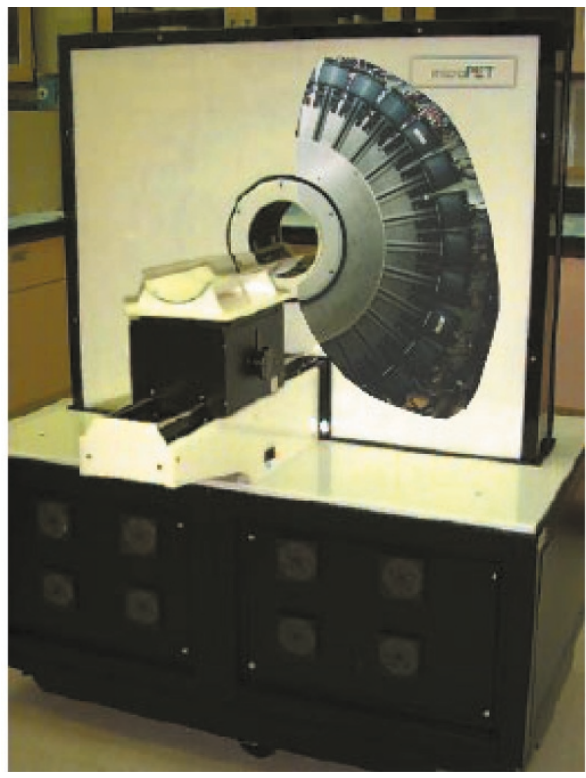

Photograph of the microPET scanner designed at UCLA, Los Angeles, CA, USA. The scanner is capable of imaging small animals such as mice in 30-60 min with a spatial resolution of $\sim 2 \mathrm{~mm}$ in each axis (8 $\mathrm{mm}^{3}$ ). The scanner uses an $8 \times 8$ array of $2 \times 2 \times 10 \mathrm{~mm}^{3}$ lutetium oxyorthosilicate crystals. The ring diameter is $17.1 \mathrm{~cm}$. The transaxial and axial fields of view are 17.1 and $1.8 \mathrm{~cm}$, respectively.

\section{Supplementary Figure 4}

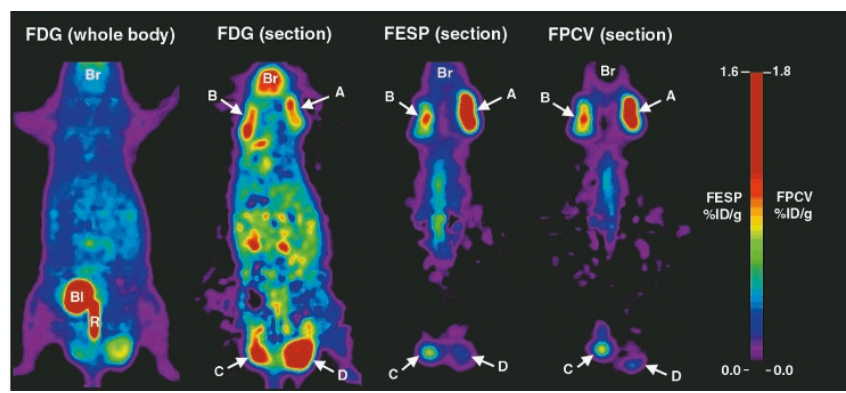

Imaging bicistronic gene expression. MicroPET imaging of the pCMVD2R-IRES-tkm C6 tumors in a nude mouse. Three C6 cell lines stably transfected with pCMV-D2R-IRES-sr39tk (labeled A, B, C) and the C6 parental cell line (labeled $D$ ) were injected into four separate sites in a single mouse. After 10 days, when each tumor was at least $6 \mathrm{~mm}$ in diameter, the mouse was imaged with 2-[18 $\mathrm{F}]$-2-fluoro-deoxyglucose (FDG), followed $24 \mathrm{~h}$ later with $\left[{ }^{18} \mathrm{~F}\right]-$ fluoroethylspiperone (FESP), followed $24 \mathrm{~h}$ later with 8-[18 $\mathrm{F}]$-fluoropenciclovir (FPCV). The FDG (whole body) image represents the average of all coronal (horizontal) planes and therefore the four tumors are not well visualized. The FDG (section) image taken from a set of planar images passing through all four tumors shows accumulation of FDG in all four tumors. Correlated signal intensity is observed between the FESP and FPCV images for each tumor. There is a high correlation between FESP and FPCV microPET signals. Br, Brain; BI, bladder; R, rectum; \%ID/g, percent injected dose per gram of tissue. 